\title{
Histerectomía Vaginal. Modificaciones a la Técnica
}

\author{
HOSPITAL DE SAN JUAN DE DIOS DE CALI
}

Dres.: Néstor H. Amorocho* y Luis M. Castro**

\section{INTRODUCCION}

En nuestro medio, entre las entidades Ginecológicas que requieren tratamiento quirúrgico, se presenta con mayor frecuencia el prolapso genital. Experiencia previa (1) en cirugía vaginal por prolapso en el servicio de Ginecología del Hospital de San Juan de Dios, nos mostró morbilidad elevada en la histerectomía vaginal:

$\begin{array}{lc}\text { Retención urinaria } & 29.2 \% \\ \text { Hematoma y/o absceso } & 13.3 \% \\ \text { Fístulas } & 1.3 \% \\ \text { Hemorragia y/o choque } & 0.9 \% \\ \text { Peritonitis } & 0.44 \% \\ \text { Mortalidad } & 1.3 \%\end{array}$

Este trabajo realizado doce años atrás, nos movió a investigar otros parámetros relacionados con la corrección del prolapso genital, por histerectomía vaginal y colporrafîas.

Diferentes autores (2, 3 y 4$)$ refieren porcentajes de recidiva de la incontinencia urinaria de esfuerzo (IUE) desde 10 hasta $52 \%$ después de la operación de Kelly, cuyo mérito fundamental ha sido baja morbilidad, pronta recuperación post-operatoria.

Las consideraciones anteriores nos llevaron a hacer modificaciones a la técnica tradicional, efectuando histerectomía intrafacial, dejando cúpula parcialmente abierta, fijada en forma diferente y

* Jefe Servicio de Ginecología.

** R-3 Gineco-Obstetricia - Univalle. efectuando uretrocistopexia por vía vaginal, reemplazando la plicatura de uretra y vejiga y los puntos de Kelly en el cuello vesical.

Como ya lo habíamos notado (1), la evolución de la cirugía del prolapso, tiende cada vez más a la histerectomía, frente a otras técnicas reparativas conservadoras.

De acuerdo con lo anterior, hemos querido buscar una técnica que reúna las condiciones de corrección adecuadas del prolapso y a la vez corrija la IUE, cuando está presente.

Los objetivos (4) al realizar una cirugía para corregir la IUE, deben contemplar:

- Elevar el cuello vesical y la uretra proximal para que recuperen su posición anatómica.

- Impedir que el cuello vesical se haga infundibuliforme.

- Aumentar la longitud funcional y anatómica de la uretra, incrementando la presión de cierre y la resistencia uretral.

\section{MATERIAL Y METODOS}

Se realizó revisión retrospectiva de 100 historias clínicas del total de pacientes intervenidas -Histerectomías Vaginales- en el servicio de Ginecología del Hospital de San Juan de Dios de Cali entre los años de 1981 a 1986.

La totalidad de las pacientes fueron operadas por el mismo cirujano con las modificaciones a la técnica que se describirán más adelante. 
Se diseñó un protocolo para tabular los siguientes datos:

Edad, diagnóstico del grado del prolapso, presencia o no de IUE, número de controles post-operatorios, recidiva de síntomas, hallazgos al examen ginecológico en el control post-operatorio, existencia de queja sobre función sexual, paridad y tipo de anestesia.

Como norma del Servicio toda paciente que ingresa debe tener citología vaginal reciente y evaluación prequirúrgica por el Servicio de Medicina Interna cuando su edad sea igual o mayor de 40 años.

En forma rutinaria se realiza preparación de colon con enemas evacuantes y de vagina con duchas con yodopovidona.

Al día siguiente de la cirugía, 24 horas postoperatorio, se realiza pinzamiento intermitente de la sonda vesical.

Se retira la sonda al 2o. día, 48 horas post-operatorio, verificando que no haya retención urinaria.

Se da de alta a la paciente después de tres días de estancia, 72 horas en el hospital.

Todas las pacientes se controlan en la consulta externa dentro de las cuatro semanas siguientes a la cirugía.

La elección del tipo de anestesia siguiendo la clasificación ASA, está a cargo del anestesiólogo.

La técnica quirúrgica es básicamente la histerectomía vaginal introducida por Heaney (5) desde 1934, con elevación del cuello vesical suspendiéndolo de la fascia pubovesicovaginal, adyacente a las ramas isquiopúbicas de la pelvis. Las modificaciones introducidas son las siguientes.

Evacuación de la vejiga con sonda Folley No. 18 a drenaje desde el comienzo de la intervención, consiguiendo un mayor descenso del útero y campo operatorio más amplio.

Uso de la valva de Auvard, permitiendo colaboración más efectiva del ayudante, sin sacrificar amplitud en el campo operatorio.
Después del corte circular sobre el cervix, la mucosa se separa de la aponeurosis recto-vaginal, con tijeras en lugar de disección roma y de igual manera se trata la aponeurosis vésico-vaginal.

Después de penetrar al fondo de saco posterior, se hace pinzamiento sección y ligadura de ligamentos útero-sacros, individualmente, lo más cercano posible a su inserción en el útero (ver ilustraciones Fig. 1). -E1 material de sutura utilizado, será siempre absorbible, catgut-. Se conservan los catguts reparados para posterior uso en el cierre de la cúpula o bóveda vaginal.

Pinzamiento sección y ligadura de ligamentos cardinales o de Mackenrodt; igualmente se conservan los reparos de catgut para posterior uso en cierre y mejorar el diafragma pélvico (ver ilustraciones Fig. 2). Es importante la identificación pinzamiento y sección por separado de los ligamentos úterosacros y de Mackenrodt (ver ilustraciones Fig. 2). Una vez pinzados, seccionados y ligados los pedículos superiores (ligamentos redondo, útero-ovárico y trompa) se conservan los catgut reparados para suspensión y cierre parcial de la cúpula vaginal.

Los ligamentos de Mackenrodt se aproximan $y$ afrontan uno a otro constituyendo una hamaca del piso pélvico (ilustraciones Fig. 3).

La cúpula vaginal se suspende a los ligamentos útero-sacros y pedículos superiores fijándolos al círculo donde se practicó la circuncisión del cuello uterino de la siguiente forma:

La cúpula semeja el tablero de un reloj. Fijando el ligamento útero-sacro derecho a las 7 y a las 11 , y el izquierdo a la 1 y a las 5 ; el pedículo superior derecho a las 9 y el izquierdo a las 3 (ver ilustraciones Fig. 3); al anudarlos se afrontan los bordes de la cúpula en forma horizontal siguiendo el eje y la disposición anatómica de las paredes de la vagina (ver ilustraciones Fig. 4).

La corrección de la incontinencia urinaria se efectúa como ya se dijo, corrigiendo el ángulo vésico-uretral, suspendiendo el cuello vesical de la facia pubo-vesical. El cisto-cele se invagina con puntos de seda que toman la facia y músculo pubo-coxigeo de ambos lados.

Consideramos importante, para mejor resultado, hacer colpoperineorrafia y rafia de elevadores del ano en todos los casos. 


\section{ILUSTRACIONES DE LA TECNICA}

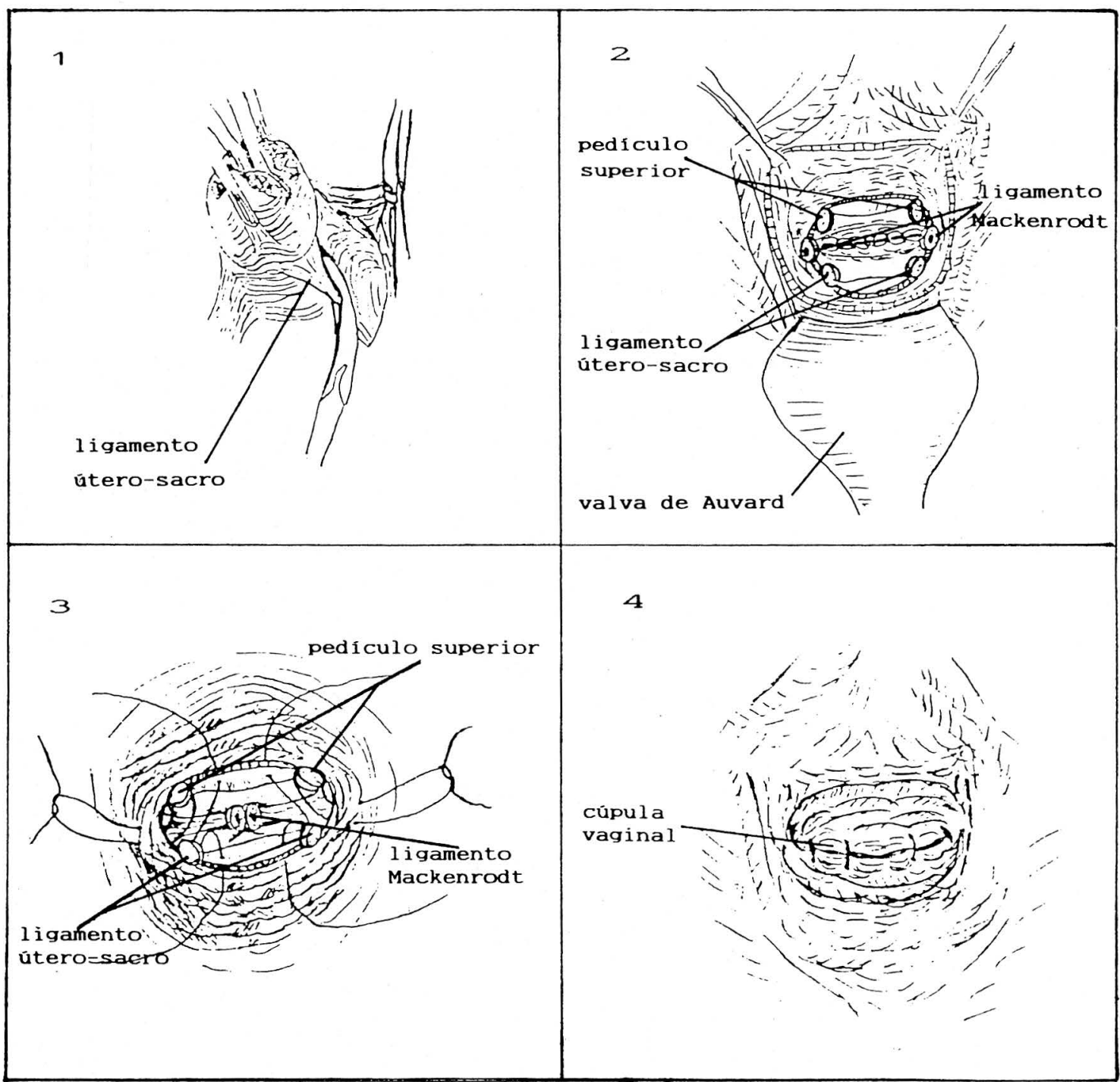

\section{RESULTADOS Y DISCUSION}

\section{Anestesia}

El tipo de anestesia utilizado en las pacientes motivo de estudio fue 46 anestesia general y 54 conductiva. El análisis no muestra ninguna incidencia en morbilidad intra o post-operatoria.

\section{Edad}

El $53 \%$ de las pacientes tenían 50 o más años; el $31 \%$ se encontraban entre los 40 y 49 años y el
$16 \%$ restantes fueron menores de 40 años. Las edades extremas fueron 29 y 85 años - Gráfico 1.

Gráfico 2 presenta el análisis del grado de prolapso genital en relación con la edad por grupos.

El prolapso grado IV se presentó en el $22 \%$ de las pacientes; $20 \%$ correspondieron a edad de $50 \mathrm{y}$ más años.

El $47 \%$ de los casos estudiados correspondió a pacientes con prolapso genital grado III, que analizadas en relación con la edad mostraron que el $40 \%$ tenían edad mayor de 40 años. 
GRAFICO 1

\section{DISTRIBUCION POR GRUPOS DE EDAD}

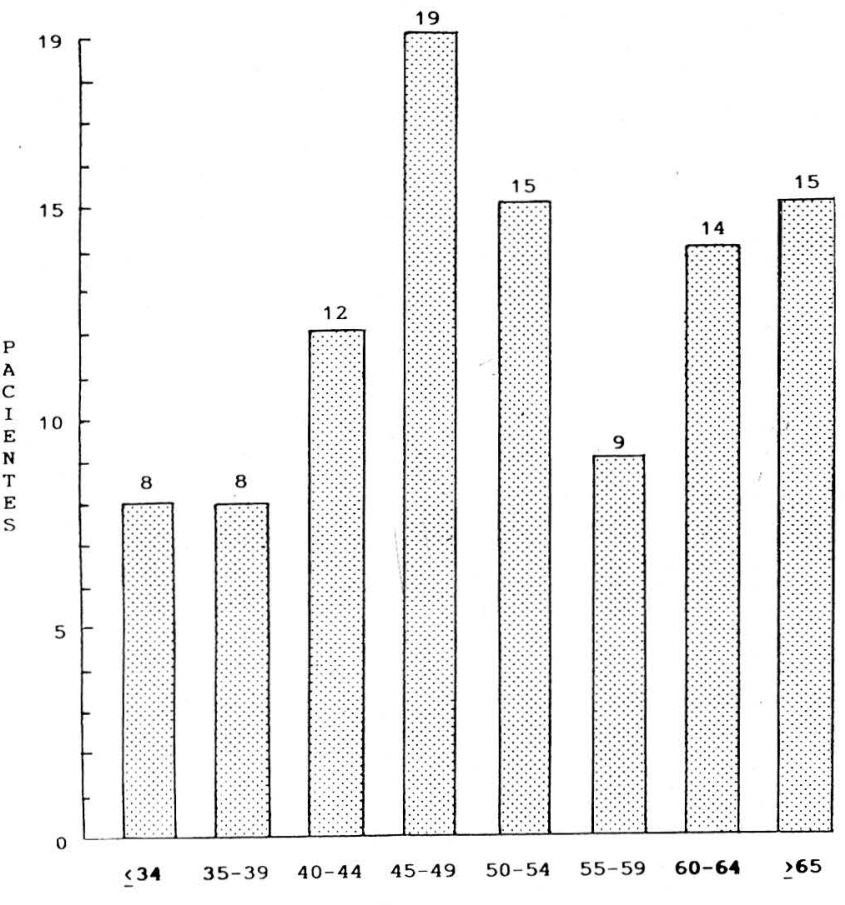

GRAFICO 2

PROLAPSO POR GRUPO DE EDAD

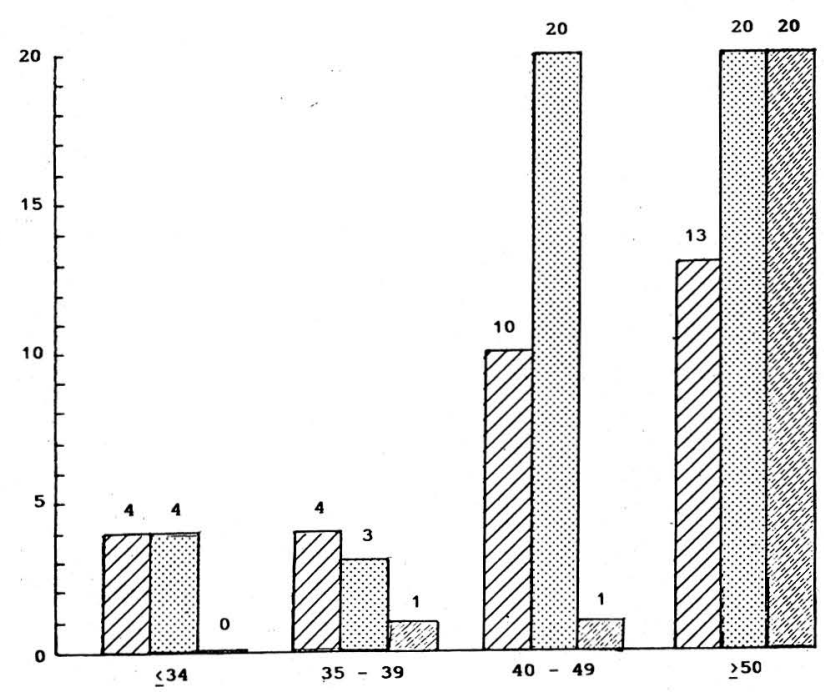

Q7 Prolapso Genital Grado 11

Prolapso Genital Grado 111

Prolapso Genital Grado IV
Se encontró prolapso genital grado II en el $31 \%$ de las pacientes, de las cuales el $23 \%$ correspondió a edad por encima de los 40 años.

En el presente estudio, la severidad del prolapso genital (medida por el grado correspondiente II - IV), guarda relación directa con la edad.

Vemos como $84 \%$ de las pacientes analizadas corresponden a edades por encima de los 40 años, frente a $16 \%$, cuyas edades están por debajo de los 40 años.

\section{Paridad}

De las 100 historias clínicas, motivo de estudio, se encontró que en 13 no estaba consignado el dato de paridad. Por tanto, se analizó la paridad en 87 pacientes. partos.

El $57.5 \%$ (50 pacientes), tenían 5 y más

El $32.2 \%$ ( 28 pacientes) tenían entre 3 y 4 partos; 8 pacientes, correspondientes a $9.15 \%$ presentaron paridad entre 1 y 2 partos.

Se encontró una paciente nulípara.

Este análisis se muestra en el Gráfico 3.

\section{Prolapso y Paridad}

De las 100 historias clínicas, motivo de estudio, como se anotó anteriormente, 13 no tenían consignado el dato de paridad y una era nulípara. En consecuencia para hacer el análisis porcentual se tomó la cifra de 86 pacientes correspondientes al $100 \%$, para la relación prolapso-paridad: Gráfico 4

El $58 \%$ de las pacientes con prolapso presentaron paridad de 5 y más partos y el $42 \%$ correspondieron al grupo de paridad de 1 a 4 partos.

E1 prolapso genital IV se encontró en el 20.9\% de las pacientes analizadas. De esta cifra porcentual el $15.1 \%$ corresponde a mujeres que tenían 5 o más partos. La paridad, como se muestra, guarda relación directa con el prolapso genital grado IV, en el presente estudio. 


\section{GRAFICO 3}

DISTRIBUCION POR GRUPO DE PARIDAD

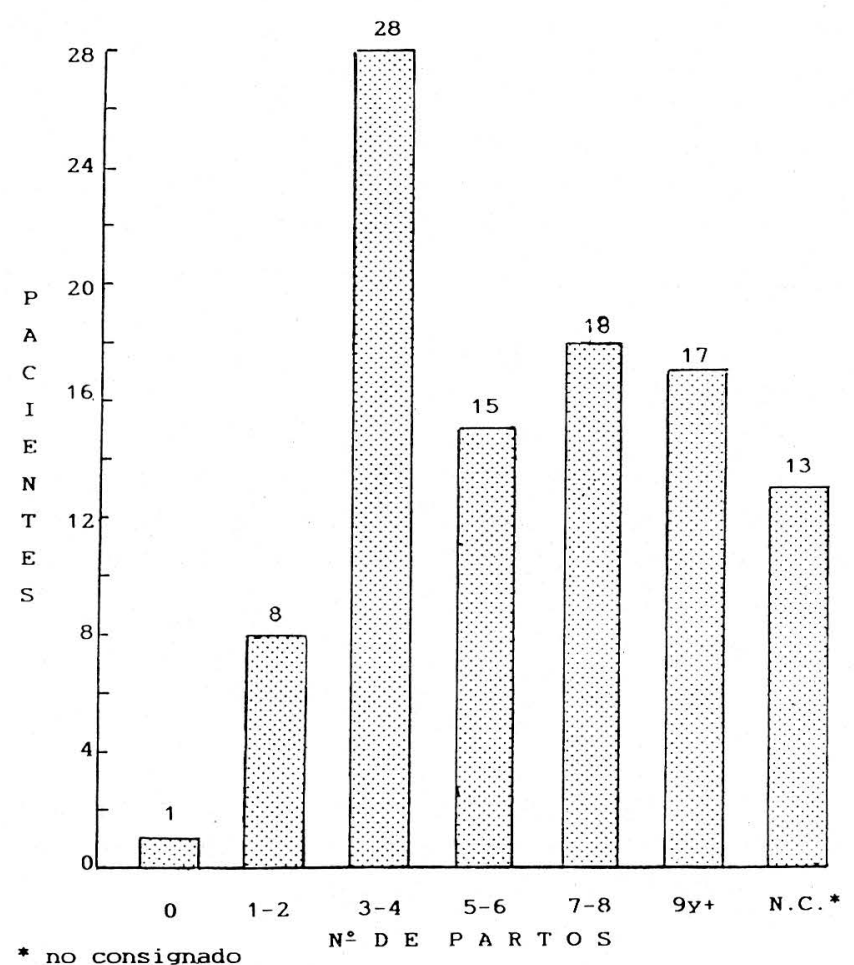

El $46.5 \%$ de las pacientes, presentaron prolapso genital III y de estas el $27.9 \%$, se encontraban en el grupo de paridad de 5 y más partos. Estas cifras porcentuales, significativamente estadísticas que correlacionan el prolapso genital grado III con la mayor paridad.

$32.6 \%$ de las pacientes tenían prolapso genital II, sin encontrarse diferencia estadística significante entre los grupos de paridad de 1 a 4 partos y 5 y más partos.

\section{Incontinencia Urinaria y Prolapso}

El dato relativo a incontinencia urinaria de esfuerzo, IUE, se encontró consignado en $86 \%$ historias clínicas, distribuido así: IUE en $71.7 \%$ de las pacientes y ausencia del síntoma en $28.3 \%$ de los casos.Gráfico 5.

El prolapso genital III guarda relación directa con IUE, $36.5 \%$ de las pacientes estudiadas, frente a $27 \%$ de las pacientes con prolapso genital II y únicamente se asoció el prolapso genital IV con IUE en el $8 \%$.

En conclusión, IUE en nuestro estudio se encontró en el $63.5 \%$ de las pacientes con diagnóstico de prolapso genital II o III.

En el prolapso genital IV la IUE no es motivo primordial de consulta, de acuerdo con nuestro estudio, como sí lo encontramos en el prolapso genital II y III.

\section{GRAFICO 4}

\section{GRADO DE PROLAPSO POR GRUPOS DE PARIDAD}

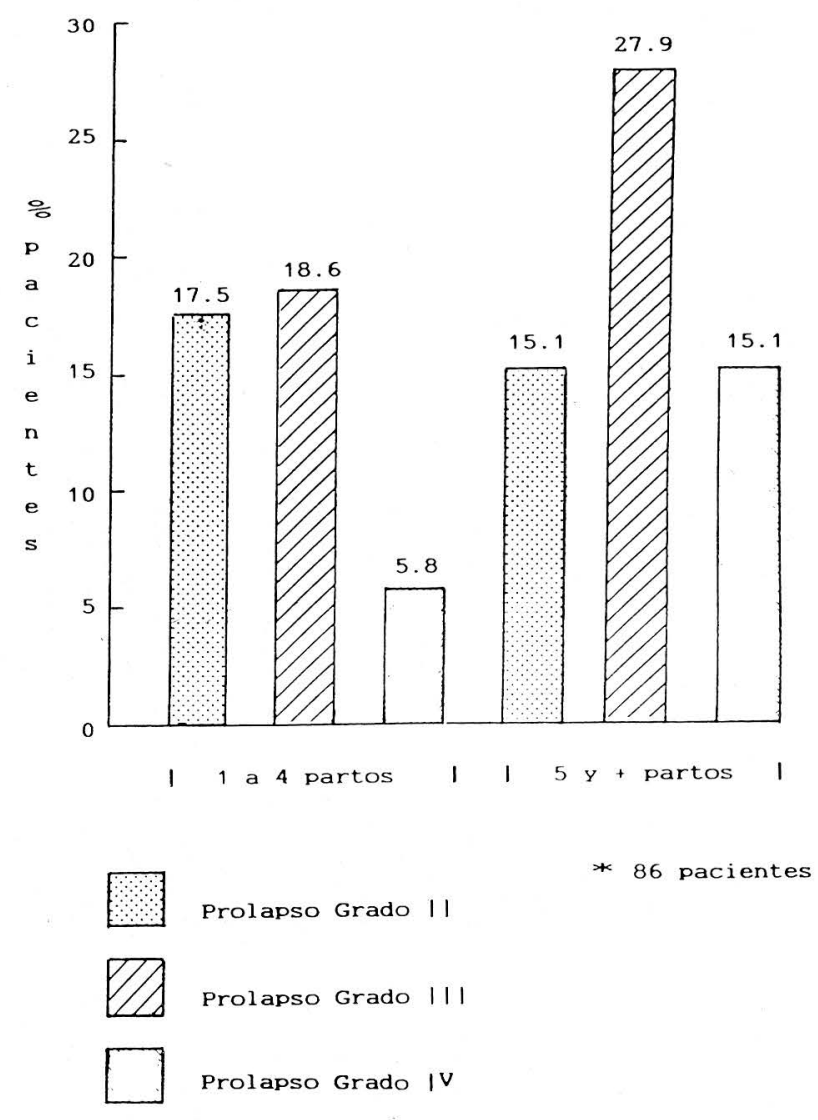




\section{Control Post-operatorio}

El Gráfico 6 muestra los datos relativos al control post-operatorio.

\section{GRAFICO 5}

\section{PROLAPSO E INCONTINENCIA URINARIA}

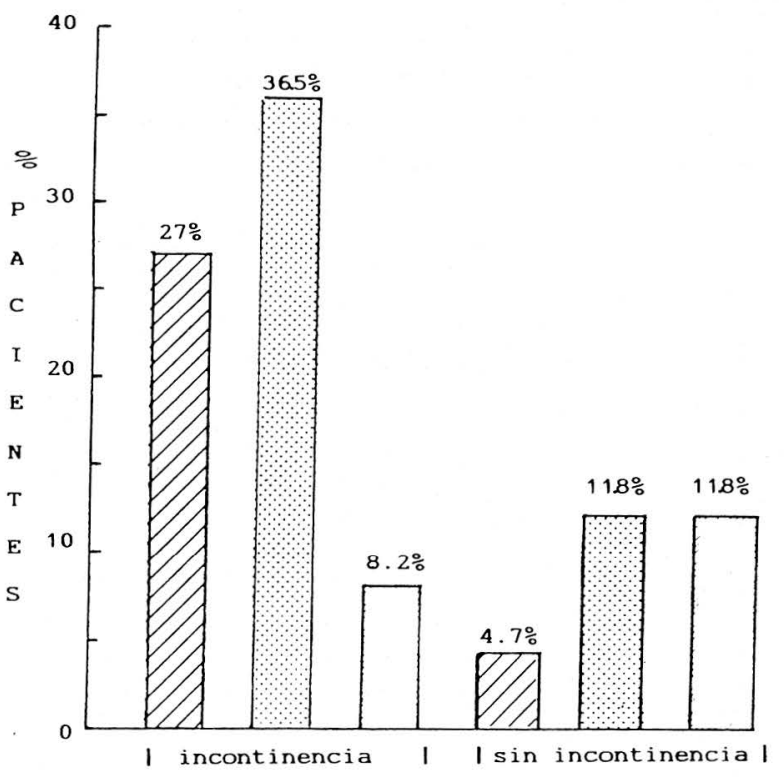

PA

Prolapso Grado II

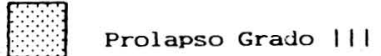

* 86 pacient

Prolapso Grado IV

CUADRO 1

MORBILIDAD
INFECCION URINARIA

HEMATOMA DE CUPULA

INFECCION AREA QUIRURGICA

RECIDIVA DE SINTOMAS
PACIENTE

3 PACIENTES*

4 PACIENTES

11 PACIENTES
3 PACIENTES

\section{GRAFICO 6}

\section{CONTROLES POSTOPERATORIOS}
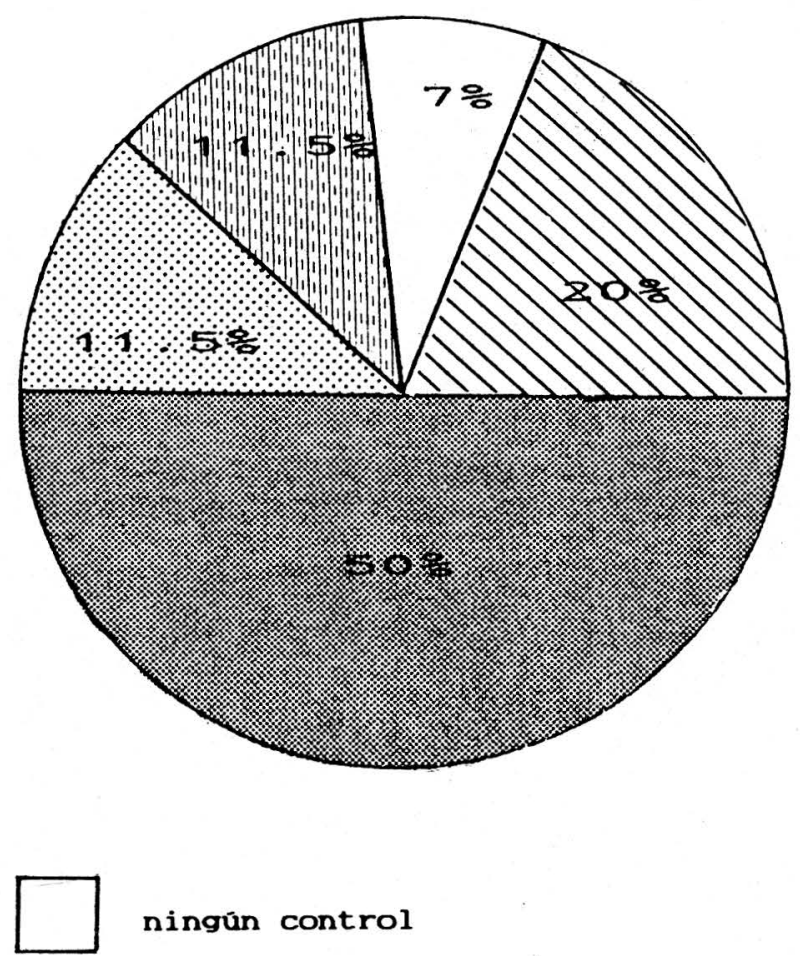

ningún control

1 control

2 controles

3 controles

4 controles

Durante el primer mes de la cirugía se efectuó control en el $50 \%$ de los casos.

$20 \%$ de las pacientes asistieron a dos controles, a tres controles $11.5 \%$, cuatro y más controles $12 \%$ y no asistieron a control el $7 \%$ de las pacientes operadas.

\section{Morbilidad}

Se presentó morbilidad post-operatoria en $11 \%$ de las pacientes. 
Una paciente presentó infección urinaria, tres pacientes hematoma de cúpula, de las cuales una se infectó; cuatro pacientes mostraron infección en área quirúrgica y recidiva de síntomas en tres pacientes, ver Cuadro 1.

\section{COMENTARIOS}

La cirugía del prolapso genital frente a parámetros encontrados en el estudio presente tales como edad y paridad, ratifica cómo la evolución del tratamiento quirúrgico tiende cada vez más a la histerectomía, frente a otras técnicas reparativas conservadoras (1); por tanto los cirujanos pélvicos competentes, deben poder realizar de $25 \%$ a $50 \%$ de las histerectomías, por vía vaginal (9).

Prolapso genital e incontinencia urinaria de esfuerzo (IUE) se encuentran frecuentemente asociados $(3,4)$ y nosotros encontramos presente la IUE en $71.7 \%$ de las pacientes, que consultaban a la vez por prolapso.

Se han modificado e ideado una gran cantidad de técnicas tendientes a corregir la IUE, desde cuando Kelly describió la plicatura de la Uretra, técnica que revisada por otros autores muestra recidivas hasta de $52 \%(2,3,4)$ y otras técnicas descritas $(6$, $7)$ indican incapacidad para corregir este trastorno.

Creemos que la vía vaginal como tratamiento quirúrgico de la IUE asociada con prolapso genital, con tinúa teniendo vigencia.

La virtud de la cirugía por vía vaginal radica en el hecho de poder realizar varios procedimientos: Corregir la IUE, el prolapso genital obteniendo con poca morbilidad, un resultado estético y funcional excelentes (8).

La morbilidad en nuestro estudio es baja y el resultado no se alteró; sólo tuvimos recidiva de síntomas en $3 \%$ de los casos.

Es posible que el éxito del tratamiento quirúrgico radique principalmente en la selección cuidadosa de las pacientes y el uso de una técnica quirúrgica depurada.

Nos identificamos con otros autores (9) que hacen recomendaciones iguales a las que nosotros seguimos en la técnica que presentamos.
Es muy importante colocar la paciente en la posición adecuada; los ayudantes deben tener acceso a la vagina lo mismo que el cirujano.

Obtenemos campo quirúrgico más amplio, cuando desde el comienzo de la intervención colocamos sonda vesical a drenaje. En el mismo sentido usamos la valva vaginal de Auvard. Otros autores (9) consideran importante el espéculo de succión (valva con tubo de aspiración) para mantener seco el campo e igual que ellos creemos que en una Histerectomía vaginal bien hecha, la hemorragia típica no debe pasar de 200 a $300 \mathrm{ml}$. Aunque no lo hacemos de rutina, es recomendable que el cirujano utilice una lámpara frontal como la que usaba el Dr. Nichols (9).

Para disminuir la aparición de granulomas, tan molestos en el post-operatorio para paciente y médico, pensamos que debe usarse material absorbible (catgut) y sólo usamos seda para la uretro-cistopexia.

La técnica que presentamos, para cierre y suspensión de la cúpula vaginal, preserva la longitud, el eje y la configuración anatómica de la vagina.

\section{RESUMEN}

Se revisaron 100 historias clínicas correspondientes a histerectomías vaginales realizadas con las modificaciones a la técnica tradicional en el servicio de Ginecología del Hospital de San Juan de Dios de Cali.

Se presenta en éste trabajo una técnica quirúrgica que incluye varias modificaciones a los procedimientos clásicos tradicionalmente conocidos, las cuales se describen a continuación:

1. Evacuación de la vejiga al inicio de la cirugía y se continúa con la sonda a drenaje, obteniende mayor descenso del útero y campo operatorio más amplio.

2. Uso de la valva de Auvard: campo operatorio más amplio sin intervención del ayudante, permitiendo la colaboración de él activamente en la operación.

3. Se identifican, pinzan y seccionan por separado los ligamentos cardinales o de Mackenrodt 
y los ligamentos útero-sacros y se dejan reparados. Los ligamentos de Mackenrodt se aproximan y afrontan uno a otro constituyendo una hamaca del piso pélvico.

4. Suspensión de la cúpula: los ligamentos úterosacros se fijan a las horas 1 y 5 y a las 11 y 7 del círculo que queda al circuncidar el cuello, correspondiendo al canal vaginal; en tanto que los pedículos superiores, que al ser reparados contienen cada uno el ligamento redondo, la trompa y el ligamento útero-ovárico, se llevan a las horas 3 y 9 .
Esta técnica de tipo intrafacial preserva la configuración anatómica, la longitud y el eje de la vagina. nima.

La morbilidad igual que otros autores, es mí-

Creemos que la uretro-cisto-pexia, llevando los puntos a la facia pubovesical y afrontar las estructuras, restablece el ángulo vésico-uretral corrigiendo la incontinencia urinaria.

\section{BIBLIOGRAFIA}

1. HENAO, F.; RESTREPo, H.; AMOROCHO, N.: Girugía del prolapso genital. Hospital de San Juan de Dios de Cali, 19581964. Revista Colombiana de Obstetricia y Ginecología. Vol. XXVII No. 6, 354-362, 1976.

2. HODGKINSON, P.: Stress urinary incontinence. Am. Journal of Obstetrics and Gynecology. 108, 1141, Dic. 1, 1970.

3. GREEN, T.H.: Urinary stress incontinence: differential diagnosis, pathophysiology and management. Am. Journal of Obstetrics and Gynecology 122, 368, 400, 1975.

4. GOMEZ, P.; LOMANTO, A.: Operación de Kelly. Revista Colombiana de Obstetricia y Ginecología. Vol. XXXIV No. 1, 1983.
5. STANTON, S.L.: Cirugía de la incontinencia urinaria. Temas Actuales Ginecología y Obstetricia. Vol. I 79-104, 1978.

6. DURFEE, R.: Simposio de prolapso genital. Clinics Obstetrics and Gynecology 997, 1966. Edit. Interamericana.

7. BELL, T.: Gynecology Surgery and Urology. Sección IV y V. 2nd edition. The C V Mosby Co. 1963.

8. DICKER, R., et al.: Complication of abdominal and vaginal histerectomy in USA. Am. Journal of Obstetrics and Gynecology. Vol. 144 No. 7, 841, 1982.

9. BARTER, R.: Histerectomía Vaginal. Clínicas Obstétricas y Ginecológicas. No. 4, 1982. Edit. Interamericana. 